\title{
Synchronous with Your Feelings: Sensorimotor $\gamma$ Band and Empathy for Pain
}

\author{
Viviana Betti, ${ }^{1,2}$ Filippo Zappasodi, ${ }^{3}$ Paolo Maria Rossini, ${ }^{4,5}$ Salvatore Maria Aglioti,, 6 and Franca Tecchio ${ }^{3,5}$ \\ ${ }^{1}$ Associazione Fatebenefratelli per la Ricerca, Ospedale Fatebenefratelli, 00186 Rome, Italy, ${ }^{2}$ Fondazione Santa Lucia, Istituto di Ricovero e Cura a Carattere \\ Scientifico, 00142 Rome, Italy, ${ }^{3}$ Istituto Scienze e Tecnologie della Cognizione, Consiglio Nazionale delle Ricerche, 00185 Rome, Italy, ${ }^{4}$ Clinica Neurologica, \\ Università Campus Biomedico, 00128 Rome, Italy, ${ }^{5}$ Casa di Cura San Raffaele, 03043 Cassino, Italy, and San Raffaele Pisana, Istituto di Ricovero e Cura a \\ Carattere Scientifico, 00163 Rome, Italy, and 'Dipartimento di Psicologia, Sapienza Università di Roma, 00185 Rome, Italy
}

Neuroscience studies on the social sharing of observed or imagined pain focused on whether empathic pain resonance is linked to affective or sensory nodes of the pain matrix. However, empathy, like other complex cognitive processes, is inherently linked to the activation of functional networks rather than of separate brain areas. Here, we used magnetoencephalography (MEG) to explore the relationship between empathy and functional coupling of neuronal activity in primary somatosensory (SI) and motor (MI) cortices. MEG recording was performed while healthy participants observed movie-clips depicting the static hand of a stranger model, the same hand deeply penetrated by a needle, or gently touched by a Q-tip. Subjects were asked to rate the movie-derived sensations attributed to self or to the model. For each type of clip observation, we analyzed spectral power and coherence values in $\alpha, \beta$, and $\gamma$ frequency bands. While spectral power indexes separate neural activity in SI and MI, coherence values index functional cross-talk between these two areas. No power changes of SI or MI sources were induced by observation conditions in any of the frequency bands. Crucially, $\gamma$-band coherence values were significantly higher during needle-in-hand than touch and static hand observation and correlated with self-and otherreferred pain ratings derived from needle-in-hand movies observation. Thus, observation of others' pain increases neuronal synchronization and cross-talk between the onlookers' sensory and motor cortices, indicating that empathic resonance relies upon the activity of functional networks more than of single areas.

\section{Introduction}

A large variety of painful experiences, ranging from physical pain perception (e.g., a wound) (Tracey and Mantyh, 2007) to social rejection (Eisenberger et al., 2003) activates a widely distributed brain network, referred to as the "pain matrix" (Melzack, 1999). The ability to understand others by sharing their intentions, emotions, and feelings, a distinctive feature of empathy (Preston and de Waal, 2002), is mapped on largely overlapping neural structures (Hein and Singer, 2008).

Previous studies focused on whether vicarious pain is mapped on affective or sensory nodes of the pain matrix (Hein and Singer, 2008). However, empathy for pain, like higher-order cognitive functions, may be based on the functional interaction of neuronal assemblies distributed within and across different specialized brain regions (Varela et al., 2001; Fries, 2005, 2009). Indeed, mounting evidence indicates that rhythmic neural oscillations and their synchronization provide indices of dynamic and flexible communication between and within the cerebral networks underlying a specific behavior (Fries, 2005). Synchronized oscil-

\footnotetext{
Received June 12, 2009; revised July 25, 2009; accepted Aug. 12, 2009.

The financial contribution of Ministero Istruzione Università e Ricerca (PRIN) and Ministero Sanità, Italy is gratefully acknowledged. Thanks are due to Matilde Ercolani for her excellent technical assistance.

Correspondence should be addressed to either Viviana Betti or Salvatore M. Aglioti, Fondazione Santa Lucia, Via

Ardeatina 306,00142 Rome, Italy, E-mail: viviana.betti@gmail.com or salvatoremaria.aglioti@uniroma1.it.

V. Betti's and F. Zappasodi's present address: Università "G. d'Annunzio," 66013 Chieti, Italy.

D01:10.1523/JNEUROSCI.2759-09.2009

Copyright $\odot 2009$ Society for Neuroscience ～0270-6474/09/2912384-09\$15.00/0
}

latory activity in the $\alpha, \beta$, and $\gamma$ frequency bands is associated to a number of functions ranging from working memory and mental imagery to selective attention, sensorimotor integration and perceptual awareness. Moreover, disrupted oscillatory activity seems associated to neuropsychiatric disorders (e.g., schizophrenia) characterized by dysfunctional cognition and behavior (Uhlhaas et al., 2008). In particular, studies support the role of $\gamma$-frequency band $(>30 \mathrm{~Hz})$ synchronization as a mechanism underlying the dynamic construction of the large-scale neuronal networks involved in object representation (Tallon-Baudry and Bertrand, 1999), binding of visual features (Singer and Gray, 1995; Engel and Singer, 2001), selective attention and memory (Womelsdorf and Fries, 2006; Jensen et al., 2007; Doesburg et al., 2008), sensorimotor integration (Szurhaj et al., 2006), and even very complex processes such as the "aha!" component of cognitive insight (Jung-Beeman et al., 2004; Sheth et al., 2009). Relevant to the present study is a recent MEG report showing that pain-induced $\gamma$ oscillations in primary somatosensory cortices are specifically related to the subjective pain experience instead than to the objective attributes of painful stimuli such as their actual intensity (Gross et al., 2007). Somatosensory evoked potential (SEP) and transcranial magnetic stimulation (TMS) studies show that primary somatosensory (SI) (Bufalari et al., 2007) and motor (MI) (Avenanti et al., 2005, 2006, 2009; Fecteau et al., 2008; Minio-Paluello et al., 2006, 2009) cortices are involved in the direct observation of pain stimuli delivered to stranger individuals. However, no studies have so far investigated whether empathic pain mapping is linked to the construction of a functional SI-MI network. 
Here, we explored for the first time the possible link between neuronal synchronization and empathic sharing of others' pain by obtaining magnetoencephalographic (MEG) indices of separate neural activity in primary somatosensory (SI) and motor (MI) cortices (using spectral power) and of functional cross-talk between these two areas (using sensorimotor coherence) in $\alpha, \beta$, and $\gamma$ frequency bands while subjects observed painful or innocuous stimuli delivered to the hand of stranger model. Moreover, to explore whether attentional, sensory, and motor states of the onlooker influence the emergence of a functional sensorimotor network, subjects were asked to stay relaxed, to perform a motor task or to perceive a non-noxious electrical stimulation during the observation of video-clips.

\section{Materials and Methods}

\section{Subjects}

Twelve healthy subjects ( 9 women) aged 20-25 (mean 23.64 years) were recruited for the study. All participants were right-handed according to the Edinburgh Handedness Questionnaire (mean $81 \pm 15)$ (Oldfield, 1971). They had normal or corrected-to-normal visual acuity in both eyes and were naive as to the purposes of the experiment. Participants gave their written informed consent. The procedures were approved by the Fatebenefratelli "S. Giovanni Calibita" ethics committee and were in accordance with the standards of the 1964 Declaration of Helsinki.

\section{$M E G$ recording}

Neural activity was recorded using a 28-channel system operating in a magnetically shielded room (Vacuumschmelze). The active channels (16 internal axial gradiometers and the other 12 peripheral magnetometers) were regularly distributed on a spherical surface $(13.5 \mathrm{~cm}$ of curvature radius) covering a total area of $\sim 180 \mathrm{~cm}^{2}$. The MEG system was centered on the C3 position of the 10-20 International EEG system (Jasper, 1958), in correspondence of the primary sensorimotor hand area. The electromyographic (EMG) signal from the right thumb's opponent muscle was acquired by a pair of electrodes. The active electrode was placed on the skin over the belly of the muscle and the reference electrode was placed in correspondence of the thumb metacarpophalangeal joint at a distance of $2.5 \mathrm{~cm}$ from the active one. The MEG and EMG signals were bandpass filtered $(0.48-256 \mathrm{~Hz}$, sampling rate $1000 \mathrm{~Hz})$, digitized and stored on a computer for off-line analysis.

\section{Visual stimuli}

Neuronal recording was performed during observation of video-clips depicting the following: (1) the ventral view of a still right hand ("Static"); (2) a needle penetrating the opponent muscle of the thumb of a right hand ("Needle"); (3) a Q-tip gently touching the skin area over the same muscle ("Q-tip") (see supplemental movie, available at www.jneurosci.org as supplemental material). The order of different types of video-clips was counterbalanced between subjects. The clips were presented via an LCD projector onto a screen positioned inside the magnetically shielded room in front of the subject. All the videos were represented from a first person perspective and lasted $7 \mathrm{~s}$. The order of video clips was counterbalanced between subjects. Only right body parts were presented in the videos so as to achieve complete congruency between the onlookers' hand and the body part penetrated or touched in the model. To avoid habituation, different versions of similar video-clips were used for each observation condition. To avoid activation of the motor mirror system due to the action observation (Rizzolatti et al., 2001), which may also modulate the activity of somatosensory cortices (Avikainen et al., 2002); in none of the videos was the holder of the syringe or the Q-tip visible.

\section{Experimental procedure}

During MEG recording, subjects were laying down on their right side in a comfortable bed and they were asked to stay awake and relax their muscles. The subjects were instructed to watch carefully and pay attention to the events depicted in the video-clips and to imagine that the observed body parts belonged to them. The observation condition blocks (Static, Q-tip, and Needle) were separated by a $5 \mathrm{~min}$ interval and their order was counterbalanced across subjects. During each observation condition block, MEG signals were recorded from the left Rolandic region corresponding to the primary sensorimotor hand area in three different experimental tasks where the subjects: (1) stayed relaxed without performing any task (rest); (2) performed an isometric contraction of the thumb's opponent muscle of the right hand (motor). For this task, subjects were instructed to perform the $10-20 \%$ of the maximum isometric contraction by using a device in which the pressure of a pump allows to force water into a small plastic tube until the liquid reach the level previously indicated by the experimenter. To ensure the correct execution of the motor task, subjects were instructed to check the amount of their isometric contraction which was displayed on a screen. The EMG was also checked by an experimenter. Contraction epochs of $\sim 20 \mathrm{~s}$ were separated by $5-10$ s pauses to avoid muscle fatigue. (3) Perceived a nonpainful electric stimulation of the right thumb (sensory) (applied through ring electrodes on the proximal and distal phalange, square wave pulses, and stimulus intensity slightly above the sensory threshold, with a frequency of $0.3 \mathrm{~Hz}$, duration $0.2 \mathrm{~ms}$, mean intensity of $18 \pm 5 \mathrm{~mA}$ ). No overt motor reaction was induced by the electric stimulation (Fig. 1).

In each experimental task (Rest, Motor, Sensory), subjects were asked to observe three types of clips (Static, Q-tip, Needle observational blocks). Nine different versions of each type of clip (Static, Q-tip, Needle) were used. In particular, we used: (1) three different-size and colorcontent syringes (big-red, medium-yellow, small-orange) and three different colored Q-tips (light blue, yellow, white); (2) three different penetration or touching hand points. This procedure allowed us to minimize habituation effects. Each clip lasted $7 \mathrm{~s}$. In the interval between different versions of each video-clip, a 1 s blank screen was used. MEG recording took place during the whole observation blocks. However, MEG signals acquired in the blank periods were discarded.

The stimulation site and the isometric contraction of the opponent muscle of the thumb were chosen so as to achieve complete congruency between the onlookers' stimulated hand and the body part penetrated or touched in the model. MEG recording lasted $2 \mathrm{~min}$ in rest and motor tasks. In the sensory task, an 8-min-long recording window was necessary to have the same number of segments free from artifacts due to the electrical stimulation. The interval between each task was $3 \mathrm{~min}$. The order of experimental tasks was counterbalanced across subjects.

To sum up, the MEG recording session included three observation blocks (Static, Needle, Q-tip) and three experimental tasks (rest, sensory, motor) and it lasted $\sim 60 \mathrm{~min}$.

\section{State- and trait-interpersonal reactivity measures}

State-empathy measures. State-empathy measures were obtained at the end of each observation condition block by asking subjects to evaluate the following: (1) the intensity of the sensation evoked during the observation of stimuli delivered to others (sensory, self-referred); (2) the unpleasantness of the sensation evoked during the observation of stimuli delivered to others (emotional, self-referred); (3) the intensity of the sensation supposedly felt by the model (sensory, other-referred); and (4) the unpleasantness of the sensation felt by the model (emotional, otherreferred). These ratings were obtained using 101-point visual analog scales (VAS), in which 0 indicates no sensation (intensity or unpleasantness) and 100 the maximum sensation that can be imagined.

Measures of trait empathy. Subjects were asked to complete the Italian version (Bonino et al., 1998; Albiero et al., 2006) of the Interpersonal Reactivity Index (IRI) (Davis, 1996), a 28-item self-report survey that consists of four subscales: (1) empathic concern (EC, which assesses the tendency to experience feelings of sympathy and compassion for others in need), (2) personal distress (PD, which assesses the extent to which an individual feels distress as a result of witnessing another's emotional distress), (3) perspective taking (PT, which assesses the dispositional tendency of an individual to adopt the perspective of another), and (4) fantasy scale (FS, which assesses an individual's propensity to become imaginatively involved with fictional characters and situations). Current social psychological interpretations indicate that while PD taps selforiented aspects of interpersonal reactivity, the other three subscales, namely EC, PT, and FS tap other-oriented interpersonal reactivity. 


\section{Data handling}

The first step of the analysis we have employed is a semiautomatic independent component analysis (ICA)-based procedure to identify and remove cardiac and/or ocular artifacts without rejecting the contaminated epochs. The ICA procedure, as many other blind source separation (BSS) techniques, decomposes the EEG/ MEG data into sources with independent time course on the basis of the statistical properties of the generated signal. No information about head geometry or electrode locations (Makeig et al., 2004) but only information contained in the waveform of original signals is taken into account. The main ICA assumption is that a set of statistically mutually independent sources $\mathbf{s}$ have been mixed linearly in the recorded data $\mathbf{x}$ by means of a mixing matrix $\mathbf{A}$. The aim is to recover both $\mathbf{s}$ and $\mathbf{A}$ starting from the observation of the linear mixture $\mathbf{x}=$ As. Several studies have proved its effectiveness in removing artifacts and extracting relevant activations from MEG and EEG signals (Barbati et al., 2004). However, some of the distinctive features of the electrophysiological signals are well known. Thus, a new approach, recently developed by Tecchio et al. (2007), named functional source separation (FSS), allows to enhance the separation of relevant signals by exploiting some a priori knowledge which takes into account also the information contained in original signal waveforms. With respect to the standard ICA, a modified contrast function is defined as: $F=J+\lambda R$, where $J$ is the statistical index normally used in ICA, while $R$ accounts for the prior information of the sources. According to the weighting parameter $\lambda$, it is possible to adjust the relative weight of these two aspects. Moreover, since prior information on the sources may also be described by a nondifferentiable function, the new contrast function $F$ is optimized by means of simulated annealing. This procedure does not require the use of derivatives and allows to achieve global optimization, while gradient-based algorithms usually employed in ICA only guarantee local optimization (Barbati et al., 2006). To identify the specific contribution of different neuronal sources (which are assumed to be nonindependent/noncorrelated), the FSS source-specific functional constraints are applied to the original data. Our study aimed to investigate the separate activity in SI and MI and their cross-talk by means of spectral power and sensorimotor coherence. Thus, we first identified the neuronal sources. The localization of the primary somatosensory cortex was obtained by analyzing the cortical reactivity to the sensory stimulation of the thumb, in a given time window. The identified neuronal source was named $\mathrm{FS}_{\mathrm{SI}}$. As the cortical reactivity (thought to originate from area $3 \mathrm{~b}$ ) is maximal at $\sim 24 \mathrm{~ms}$ from the stimulation of the finger, the functional constraint taking into account the "reactivity" to the stimuli was defined as follows:

$$
R_{\mathrm{S} 1}=\sum_{t_{24}-2}^{t_{24}+23}|\mathrm{EA}(t)|-\sum_{10}^{15}|\mathrm{EA}(t)|
$$

with the evoked activity EA computed by averaging source signal epochs triggered on the thumb stimulus $(t=0) ; t_{24}$ is the time point with the maximum magnetic-field power on the maximal original MEG channel $\sim 24$ ms (searched in the [20-28] ms window) after the stimulus delivery; the baseline (no response) was computed in the time interval from 10 to $15 \mathrm{~ms}$. As only one component is extracted at each time, it is possible to avoid the amplitude indeterminacy inherent to the general ICA method. Once the source which optimizes the contrast function $F$ has been obtained, the estimated solution is multiplied by the Euclidean norm of its weight vector $\boldsymbol{a}_{\mathrm{S} 1}\left(a_{\mathrm{S} 1}\right.$ such as $\boldsymbol{a}_{\mathrm{S} 1}=a_{\mathrm{S} 1} \hat{\boldsymbol{a}}_{\mathrm{S} 1}$, with $\left.\left|\hat{\boldsymbol{a}}_{\mathrm{S} 1}\right|=1\right)$. This makes possible amplitude comparisons among sources in a fixed position.
Based on a large body of literature demonstrating that the corticalmuscular coupling in the $\beta$ band is an index of the primary motor cortex involvement in the voluntary movement (i.e., Schoffelen et al., 2008), we used this parameter to identify the functional primary motor source$\mathrm{FS}_{\mathrm{MI}}$-devoted to the control of the thumb movements. The corresponding functional constraint was as follows:

$$
R_{\mathrm{M} 1}=\sum_{\omega_{\max }-\Delta_{1} \omega_{\max }}^{\omega_{\max }+\Delta_{2} \omega_{\max }} \operatorname{Coh}(\omega),
$$

where Coh is a function of frequency $\omega$, obtained for each $\omega$ as the amplitude of the cross-spectrum between the $\mathrm{FS}_{\mathrm{MI}}$ source signal and the rectified OP EMG during the isometric thumb opposition to the other fingers, normalized by the root mean square of the power spectral densities of these two signals; $\Delta_{1} \omega_{\max }\left(\Delta_{2} \omega_{\max }\right)=$ frequency point corresponding to a coherence amplitude of $50 \%$ of the maximal value between 13.5 and $33 \mathrm{~Hz}$ - called $\omega_{\max }$ - before (after) $\omega_{\max }$. As for $\mathrm{FS}_{\mathrm{SI}}, \mathrm{FS}_{\mathrm{MI}}$ was obtained by multiplying it by the Euclidean norm of its weight vector $\boldsymbol{a}_{\mathrm{M} 1}$ $\left(a_{\mathrm{M} 1}\right.$ such as $\boldsymbol{a}_{\mathrm{M} 1}=a_{\mathrm{M} 1} \hat{\boldsymbol{a}}_{\mathrm{M} 1}$, with $\left.\left|\hat{\boldsymbol{a}}_{\mathrm{M} 1}\right|=1\right)$.

Although the FSS constraints allow identifying sources on the basis of their function behavior, the field distribution they generate can be obtained by retro-projecting the source activity in the sensor space and can be used as the input for inverse-problem solution algorithms. We used the model of a single or multi-dipoles within an isotropic spherical head, and the sources were in all cases positioned in agreement with well established anatomical knowledge, i.e., $\mathrm{FS}_{\mathrm{SI}}$ in the postcentral and $\mathrm{FS}_{\mathrm{MI}}$ in the precentral walls of the $\omega$-shaped region of the Rolandic sulcus (Tecchio et al., 2007). Mean ( \pm SD) positions of the two sources were $(x=-31 \pm 6$, $y=16 \pm 14, z=91 \pm 18, \mathrm{~mm})$ and $(x=-22 \pm 6, y=20 \pm 19, z=103 \pm$ $14, \mathrm{~mm}$ ), respectively for $\mathrm{FS}_{\mathrm{SI}}$ and $\mathrm{FS}_{\mathrm{MI}}$, in the coordinate system defined on the basis of the subjective anatomical landmark. Thus, the positive $y$-axis passes through the nasion and the midpoint between the two preauricular points, the positive $z$-axis passes through the vertex perpendicular to $y$-axis, thus the positive $x$-axis is rightward. 

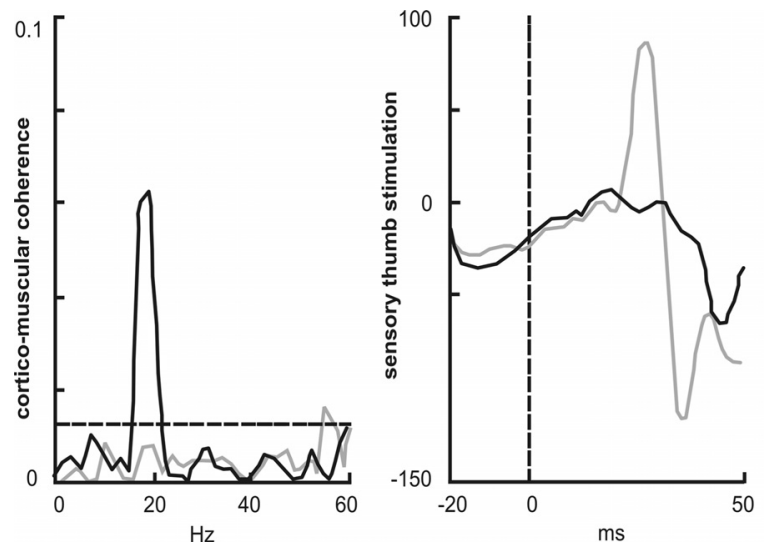

Figure 2. Functional separation of $\mathrm{FS}_{S \mid}$ and $\mathrm{FS}_{\mathrm{MI}}$ neuronal sources. The left part of the figure shows higher cortico-muscular coherence for the motor $\left(\mathrm{FS}_{\mathrm{MI}}\right)$ than the sensory source $\left(\mathrm{FS}_{\mathrm{SI}}\right)$ $\sim 20 \mathrm{~Hz}$, during the motor task. Coherence values are reported on the $y$-axis and frequency values on the $x$-axis. The horizontal stippled line indicates the significance threshold. The right part of the figure shows that, in the sensory task, the response to thumb stimulation was higher for $\mathrm{FS}_{\mathrm{SI}}$ than for $\mathrm{FS}_{\mathrm{MI}}$ source. The $y$-axis refers to the response amplitude (arbitrary unit). The $x$-axis reports the time range of the response in milliseconds. The vertical stippled line indicates the onset of the electric stimulus.

To better characterize the functional separation between $\mathrm{FS}_{\mathrm{SI}}$ and $\mathrm{FS}_{\mathrm{MI}}$, we analyzed the cortico-muscular coherence and the responsiveness to the electrical stimuli for both neuronal sources. Cortico-muscular (CMC) coherence during the isometric contraction of the thumb was much higher for MI than SI source (mean across subjects $\mathrm{CMC}_{\mathrm{MI}}=$ $0.023 \pm 0.014 ; \mathrm{CMC}_{\mathrm{SI}}=0.012 \pm 0.009$, paired sample $t$ test $\left.p=0.005\right)$ (Fig. 2, left panel). Conversely, during sensory thumb stimulation, responsiveness was much stronger for $\mathrm{FS}_{\mathrm{SI}}(52 \pm 25)$ than for $\mathrm{FS}_{\mathrm{MI}}(20 \pm$ 15 , paired sample $t$ test $p=0.037$ ) (Fig. 2, right panel).

After neuronal sources identification, across-source synchrony was directly measured for each observation condition and experimental task. Thus, the FSS procedure allowed us to investigate neuronal activity originating from separate sources, namely SI $\left(\mathrm{FS}_{\mathrm{SI}}\right)$ and $\mathrm{MI}\left(\mathrm{FS}_{\mathrm{MI}}\right)$. This neuronal activity was indexed by spectral power values in $\alpha, \beta$, and $\gamma$ frequency bands $[\alpha(8-13 \mathrm{~Hz}), \beta(13-33 \mathrm{~Hz})$ and $\gamma(33-90 \mathrm{~Hz})]$. The functional coupling between $\mathrm{FS}_{\mathrm{SI}}$ and $\mathrm{FS}_{\mathrm{MI}}$ was estimated via their spectral coherence in the $\alpha, \beta$, and $\gamma$ frequency-band and used as an index of interareal cross-talk. The coherence function was calculated for a given frequency $\omega(\alpha, \beta$, or $\gamma)$ as the square modulus of the cross-spectrum (CSD) of $\mathrm{FS}_{\mathrm{SI}}$ and $\mathrm{FS}_{\mathrm{MI}}$ normalized by the power spectral density (PSD) of the $\mathrm{FS}_{\mathrm{SI}}$ and $\mathrm{FS}_{\mathrm{MI}}$ sources. This method of functional cortical coupling has been widely used in human (Silberstein et al., 2005; Schoffelen et al., 2008; Tecchio et al., 2008) and animal studies (Young and McNaughton, 2009). Both PSD and CSD were estimated by Welch procedure (Hanning window, $512 \mathrm{~ms}$ duration, no overlap). In the Rest and Motor tasks the windows were contiguous and non-overlapping. For the sensory task we used the post-trigger intervals between 25 and $536 \mathrm{~ms}$. The $\gamma$-band synchronization was obtained as the sum of coherence values in the 33-90 $\mathrm{Hz}$ frequency interval.

\section{Statistical analysis}

Values of spectral coherence were analyzed by means of a repeated measures three-way ANOVA, with experimental task (rest, sensory, motor), frequency band $(\alpha, \beta, \gamma)$, and observational condition (Static, Needle, Q-tip) as main factors. The same type of ANOVA was performed on spectral power values, separately for $\mathrm{FS}_{\mathrm{SI}}$ and $\mathrm{FS}_{\mathrm{MI}}$ neuronal sources. Post hoc comparisons were performed by using the Newman-Keuls test. To explore any relationship between possible modulations of SI and MI neuronal activity as well as of their synchronization with subjective state- and trait-empathy measures, we performed a series of correlation analyses in which Spearman coefficients were computed.

\section{Results}

\section{Sensorimotor coherence}

$\mathrm{FS}_{\mathrm{MI}}-\mathrm{FS}_{\mathrm{SI}}$ coherence values for each experimental task, observational condition and frequency band are shown in Table 1.

The ANOVA performed on sensorimotor coherence values showed a marginal significance of the observational condition $\left(F_{(2,22)}=3.35, p=0.054\right)$ and the nonsignificance of experimental task $\left(F_{(2,22)}=1.72, p=0.20\right)$. Crucially, however, we found a significant interaction, frequency band $\times$ observational condition $\left(F_{(4,44)}=6.86, p=0.0002\right)$, which was entirely accounted for by higher sensorimotor coherence in the $\gamma$-frequency band in Needle with respect to Static ( $p=0.0002)$ and Q-tip (0.0001) observation blocks (Fig. 3).

The increase of sensorimotor coherence during pain observation was not found in $\alpha$ and $\beta$ frequency-band (all $p>0.20$ ). It is worth noting that the pain observation related enhancement of the sensorimotor coherence in the $\gamma$ frequency band is significantly higher than in $\alpha$ and $\beta$ frequency band (all $p=0.000$ ). The interaction of frequency band and experimental task was also significant $\left(F_{(4,44)}=3.06, p=0.026\right)$. Post hoc comparisons show that this effect is entirely accounted for by lower sensorimotor coherence in the $\beta$-frequency band during the Motor task with respect to Rest $(p=0.009)$. Importantly, the interaction experimental task $\times$ observational condition was not significant $\left(F_{(4,44)}=\right.$ $0.52, p=0.719)$. This pattern of results confirms that the emergence of a sensorimotor functional network is not influenced by the subjective state of the onlookers as also indicated by the lack of significance of the triple interaction experimental task $\times$ frequency band $\times$ observational condition interaction $\left(F_{(8,88)}=\right.$ $0.33, p=0.951)$.

To rule out that any involuntary motor contraction in the different observation conditions might have affected EMG and thus sensorimotor coherence, we entered rectified EMG power values in a two-way repeated ANOVA with frequency band $(\alpha, \beta$, and $\gamma$ ) and observational condition (Static, Q-tip, Needle) as main factors.

The significance of the frequency band $\left(F_{(2,22)}=261.35\right.$, $p<0.000)$ simply reflects the different spectral content of the different frequency bands. Importantly, no significant effect of observational condition $\left(F_{(2,22)}=0.09, p=0.92\right)$ or of its interaction with frequency band was found band $\left(F_{(4,44)}=0.92, p=\right.$ $0.46)$. This pattern of results rules out that any spurious effects of EMG activity influenced the specific modulation of sensorimotor coherence in $\gamma$ band during pain observation.

\section{Spectral power}

Power values for $\mathrm{FS}_{\mathrm{SI}}$ and $\mathrm{FS}_{\mathrm{MI}}$ sources in each experimental task, observational condition, and frequency band are shown in Table 2.

The ANOVA performed for $\mathrm{FS}_{\mathrm{SI}}$ source values showed a significant effect of the experimental task $\left(F_{(2,22)}=12.41 ; p=0.000\right)$, which was mainly accounted for by higher spectral power during rest with respect to motor $(p=0.000)$ and sensory $(p=0.005)$ tasks. Further, this result was band-specific as showed by the significance of experimental task $\times$ frequency band interaction $\left(F_{(4,44)}=9.99 ; p=\right.$ $0.000)$. Post hoc test revealed that this result was entirely accounted for by higher power values in $\alpha$ and $\beta$ (all $p=0.000$ ). In contrast, the comparisons between motor and sensory task was not significant (all $p>0.05)$. It is worth noting that no significant modulation of the experimental tasks was found in the $\gamma$-frequency band $(p \geq 0.29$ ). The ANOVA performed on $\mathrm{FS}_{\mathrm{MI}}$ neuronal source revealed only a trend effect of the experimental task $\times$ frequency band interaction $\left(F_{(4,44)}=2.38 ; p=0.066\right)$. In contrast, the main effect of the experimental task was not significant $\left(F_{(2,22)}=0.26 ; p=0.77\right)$. It is worth 
Table 1. Mean ( \pm SD) sensorimotor coherence values $\left(\mathrm{FS}_{\mathrm{SI}}-\mathrm{FS}_{\mathrm{MI}}\right)$

\begin{tabular}{|c|c|c|c|c|c|c|c|c|c|}
\hline & \multicolumn{3}{|l|}{$\alpha(8-12 \mathrm{~Hz})$} & \multicolumn{3}{|l|}{$\beta(13-32 \mathrm{~Hz})$} & \multicolumn{3}{|l|}{$\gamma(33-90 \mathrm{~Hz})$} \\
\hline & Static & Q-tip & Needle & Static & Q-tip & Needle & Static & Q-tip & Needle \\
\hline Rest & $0.025(0.01)$ & $0.022(0.01)$ & $0.024(0.01)$ & $0.050(0.02)$ & $0.047(0.01)$ & $0.064(0.02)$ & $0.035(0.01)$ & $0.028(0.01)$ & $0.078(0.02)$ \\
\hline Motor & $0.017(0.00)$ & $0.013(0.00)$ & $0.013(0.00)$ & $0.037(0.01)$ & $0.025(0.01)$ & $0.039(0.01)$ & $0.052(0.02)$ & $0.026(0.01)$ & $0.081(0.02)$ \\
\hline Sensory & $0.017(0.005)$ & $0.017(0.00)$ & $0.016(0.00)$ & $0.037(0.01)$ & $0.037(0.01)$ & $0.053(0.01)$ & $0.048(0.01)$ & $0.025(0.01)$ & $0.087(0.024)$ \\
\hline
\end{tabular}

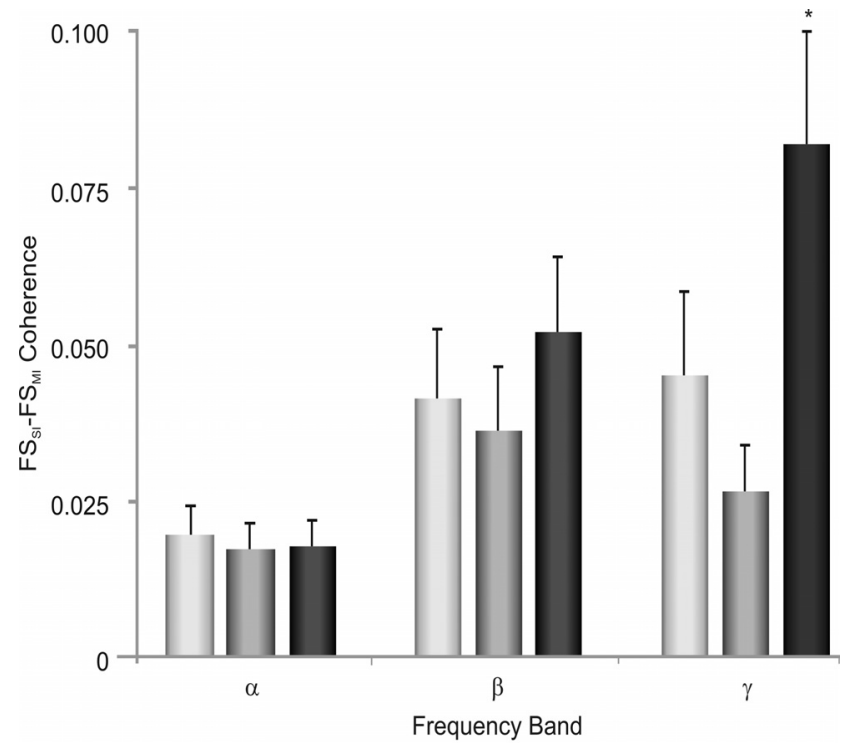

Observational Conditions: Static $\square$ Q-Tip $\square$ Needle

Figure 3. Statistical analysis of sensorimotor coherence. Columns indicate $\gamma$-band sensorimotor coherence in the different observational conditions. The asterisk $\left({ }^{*}\right)$ indicates $p<0.000$. Error bars represent SEM. Values between 0 and 1 represent the strength of coherence $(0$ indicates lack of any coherence between SI and MI).

noting that no effects of the observation condition $\left(\mathrm{FS}_{\mathrm{SI}}: F_{(2,22)}=\right.$ $0.59 ; \mathrm{FS}_{\mathrm{MI}}: F_{(2,22)}=0.10$; all $\left.p>0.05\right)$ nor of its interaction with the frequency band $\left(\mathrm{FS}_{\mathrm{SI}}: F_{(4,44)}=1.13 ; \mathrm{FS}_{\mathrm{MI}}: F_{(4,44)}=0.51\right.$; all $\left.p>0.05\right)$ were found. Further, the triple interaction experimental task $X$ frequency band $\times$ observational condition interaction was not significant $\left(\mathrm{FS}_{\mathrm{SI}}: F_{(8,88)}=1.40 ; \mathrm{FS}_{\mathrm{MI}}: F_{(8,88)}=1.30\right.$, all $\left.p>0.05\right)$.

It is worth noting that no effects of the observation condition $\left(\mathrm{FS}_{\mathrm{SI}}: F_{(4,44)}=0.59 ; \mathrm{FS}_{\mathrm{MI}}: F_{(4,44)}=0.10\right.$; all $\left.p>0.05\right)$ nor of its interaction with the frequency band $\left(\mathrm{FS}_{\mathrm{SI}}: F_{(4,44)}=1.13 ; \mathrm{FS}_{\mathrm{MI}}\right.$ : $F_{(4,44)}=0.51$; all $\left.p>0.05\right)$ were found. Further, the triple interaction experimental task $\times$ frequency band $\times$ observational condition interaction was not significant $\left(\mathrm{FS}_{\mathrm{SI}}: F_{(8,88)}=1.40 ; \mathrm{FS}_{\mathrm{MI}}\right.$ : $F_{(8,88)}=1.30$, all $\left.p>0.05\right)$.

A qualitative representation of the main results for coherence between $\mathrm{FS}_{\mathrm{SI}}$ and $\mathrm{FS}_{\mathrm{MI}}$ and the time course of the MEG signals for each source in the different observation conditions in a representative subject is reported in Figure 4. While observation of Needle brought about a clear $\gamma$ band specific increase of coherence no such effect was detected in the Static and Q-tip observation conditions. Since no difference contingent upon the subjects' experimental tasks was found, the results are reported only at rest, for the sake of simplicity.

\section{Correlation between $\mathrm{FS}_{\mathrm{MI}}-\mathrm{FS}_{\mathrm{SI}}$ coherence values in the} $\gamma$ frequency band and state- and trait-empathy measures Ratings of self- and other-oriented state-reactivity are provided in Table 3.
Spearman correlation analysis shows that sensorimotor $\gamma$-band synchronization was significantly correlated with the intensity and unpleasantness of the other-oriented $\left(r_{\mathrm{s}}=0.70, p=\right.$ $0.011 ; r_{\mathrm{s}}=0.68, p=0.015$, respectively) and self-oriented $\left(r_{\mathrm{s}}=\right.$ $0.79, p=0.002 ; r_{\mathrm{s}}=0.61, p=0.036$, respectively) sensations derived from observation of needle-in-hand movies (Fig. 5). No significant correlation with subjective ratings of Q-tip or static hand movies was found.

\section{Trait-empathy measures}

Scores on the four subscales of the Italian version of the IRI trait-empathy test were as follows: emphatic concern: 18.2 (3.6); fantasy scale: 16.2 (4.5); personal distress: 14.0 (5.9); perspective taking: 18.4 (3.7). These values are largely in accord with previous reports in samples of comparable size (Singer et al., 2004; Lamm et al., 2007). No correlation between IRI scores and $\gamma$-band coherence values was found.

\section{Discussion}

Empathy for pain studies demonstrated that empathic inference about others' pain is associated to activation of the pain matrix, i.e., the neural network that maps the first-hand experience of pain (Hein and Singer, 2008). These studies provided important clues on some of the possible variables that modulate empathy for pain (Singer et al., 2006; Avenanti et al., 2006; Cheng et al., 2007; Valeriani et al., 2008) and on whether this process impinges upon the affective (Morrison et al., 2004; Singer et al., 2004), the sensorimotor (Avenanti et al., 2005; 2006; Bufalari et al., 2007; Valeriani et al., 2008), or both components of the pain matrix (Saarela et al., 2007; Benuzzi et al., 2008; Costantini et al., 2008). Thus, the neural mapping of empathic phenomena has mainly been studied in terms of spatial changes of activity in single or multiple regions within or outside the pain matrix. It is worth noting here, that although MEG may be the ideal technique for looking at fast occurring interareas cross-talk, the only MEG study performed thus far on empathy for pain mainly demonstrates that imagination-anticipation of pain in others is mapped in the primary somatic cortex, as indexed by suppression of $10 \mathrm{~Hz}$ oscillations (Cheng et al., 2008). Yet, a critical new issue is whether, in keeping with what reported for higher-order functions, empathy is based on the dynamic interaction of neuronal assemblies that are distributed within and across different specialized brain regions rather than on signal changes within individual areas.

\section{Empathic reactivity to others' pain seems associated to the emergence of a sensorimotor functional network}

Recent functional magnetic resonance imaging evidence suggests that direct and vicarious experiences of pain may depend on patterns of functional connectivity between different areas (Zaki et al., 2007). However, the relations between interareal cross-talk and empathy for pain may be captured optimally by using hightemporal resolution techniques such as MEG. To the best of our knowledge this is the first study demonstrating that empathic reactivity to others' pain is linked to a functional network medi- 
Table 2. Mean ( \pm SD) spectral power values

\begin{tabular}{|c|c|c|c|c|c|c|c|c|c|}
\hline & \multicolumn{3}{|l|}{$\alpha(8-12 \mathrm{~Hz})$} & \multicolumn{3}{|c|}{$\beta(13-33 \mathrm{~Hz})$} & \multicolumn{3}{|c|}{$\gamma(33-90 \mathrm{~Hz})$} \\
\hline & Static & Q-tip & Needle & Static & Q-tip & Needle & Static & Q-tip & Needle \\
\hline \multicolumn{10}{|l|}{$\mathrm{FS}_{\mathrm{SI}}$} \\
\hline Rest & $7.89(0.44)$ & $7.83(0.42)$ & $7.83(0.59)$ & $7.80(0.27)$ & $7.85(0.28)$ & $7.79(0.36)$ & $7.30(0.14)$ & $7.37(0.20)$ & $7.34(0.27)$ \\
\hline Sensory & $7.49(0.35)$ & $7.53(0.30)$ & $7.41(0.40)$ & $7.54(0.25)$ & $7.62(0.25)$ & $7.48(0.27)$ & $7.29(0.23)$ & $7.31(0.19)$ & $7.29(0.20)$ \\
\hline Sensory & $7.60(0.33)$ & $7.59(0.37)$ & $7.54(0.33)$ & $7.63(0.26)$ & $7.65(0.26)$ & $7.62(0.26)$ & $7.34(0.23)$ & $7.38(0.20)$ & $7.36(0.20)$ \\
\hline \multicolumn{10}{|l|}{$\mathrm{FS}_{\mathrm{Ml}}$} \\
\hline Rest & $7.57(0.75)$ & $7.31(0.61)$ & $7.54(0.56)$ & $7.55(0.62)$ & $7.41(0.51)$ & $7.63(0.57)$ & $7.39(0.52)$ & $7.29(0.53)$ & $7.42(0.56)$ \\
\hline Sensory & $7.26(0.54)$ & $7.19(0.52)$ & $7.29(0.63)$ & $7.40(0.55)$ & $7.35(0.49)$ & $7.54(0.60)$ & $7.36(0.54)$ & $7.28(0.51)$ & $7.42(0.58)$ \\
\hline Sensory & $7.34(0.54)$ & $7.24(0.59)$ & $7.37(0.64)$ & $7.42(0.51)$ & $7.35(0.53)$ & $7.63(0.61)$ & $7.38(0.48)$ & $7.29(0.51)$ & $7.47(0.58)$ \\
\hline
\end{tabular}
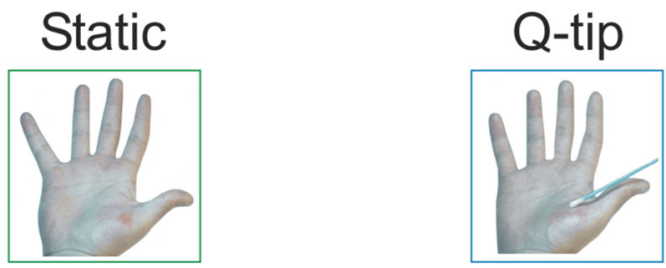

Needle
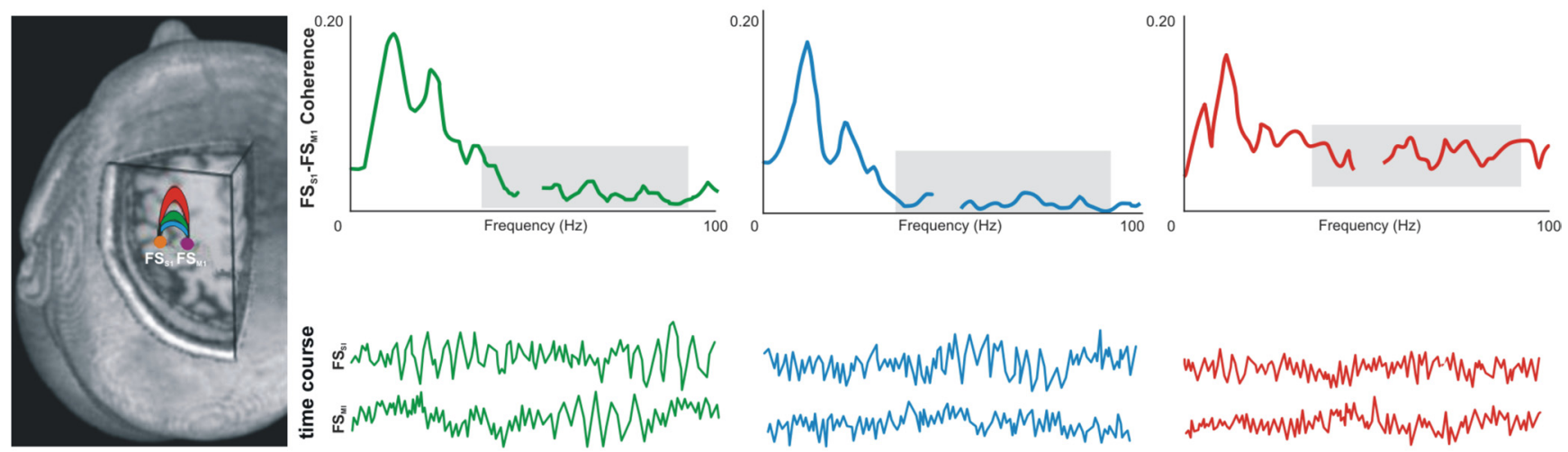

Figure 4. Sensorimotor coherence (middle row) and time course of the $\mathrm{MEG}$ signals for sensory ( $\left(\mathrm{FS}_{\mathrm{SI}}\right)$ and motor ( $\mathrm{FS}_{\mathrm{MI}}$ ) neural sources representing the thumb (lower row) in each observational condition (Static, Q-tip, Needle, upper row) in a representative subject. For the sake of simplicity, the figure represents coherence and MEG signals at rest. The gray rectangle over the sensorimotor coherence graphs indicates the $\gamma$-frequency band. The signal interruption corresponds to the $50 \mathrm{~Hz}$ line power. The panel in the left part of the figure shows a schematic cortical rendering of the somatosensory ( $\mathrm{FS}_{\mathrm{SI}}$, yellow dot) and motor ( $\mathrm{FS}_{\mathrm{MI}}$, purple dot) sources. Colored connectors depict the sensorimotor coherence in each observational condition.

Table 3. Mean ( \pm SD) subjective ratings

\begin{tabular}{|c|c|c|c|c|c|c|}
\hline & \multicolumn{3}{|l|}{ Self-oriented } & \multicolumn{3}{|c|}{ Other-oriented } \\
\hline & Static & Q-tip & Needle & Static & Q-tip & Needle \\
\hline \multicolumn{7}{|l|}{ Rest } \\
\hline Intensity & $6.67(11.23)$ & 15.92 (13.77) & $39(16.60)$ & $2.92(6.89)$ & $18.83(18.34)$ & $64(28.34)$ \\
\hline Unpleasantness & $1(2.89)$ & $2.33(5.46)$ & $45.8(18.81)$ & $1.92(6.33)$ & $3.67(8.61)$ & $68.67(29.54)$ \\
\hline \multicolumn{7}{|l|}{ Motor } \\
\hline Intensity & $3.42(5.04)$ & 14.08 (12.49) & $37.42(16.50)$ & $2.42(5.22)$ & 16.58 (16.13) & $63(29.55)$ \\
\hline Unpleasantness & $0.92(3.18)$ & $2.33(5.52)$ & 41 (19.22) & $1.75(5.19)$ & $2.92(5.55)$ & $67.83(30.41)$ \\
\hline \multicolumn{7}{|l|}{ Sensory } \\
\hline Intensity & $6.46(11.49)$ & 14.75 (13.56) & $42.71(17.81)$ & $2.92(6.72)$ & $19.96(16.07)$ & $63.92(30.08)$ \\
\hline Unpleasantness & $0.92(3.18)$ & $2.92(5.51)$ & $50.83(22.53)$ & $1.42(4.61)$ & $3.17(6.06)$ & $67.42(29.36)$ \\
\hline
\end{tabular}

ated by $\gamma$-band sensorimotor synchronization rather than to more or less independent activity in single or multiple pain matrix sections. Using MEG recording in conditions where healthy subjects observed painful or innocuous stimulation delivered to a stranger model, we found that the direct observation of others' pain induces a clear enhancement of $\gamma$-band $(33-90 \mathrm{~Hz}$ ) synchronization in the onlookers' sensorimotor system. The lack of any significant modulation in the $\gamma$-band spectral power suggests a specific role of combined rather than segregated activity of SI and MI cortices. The absence of pain observation-related coherence modulation in the $\alpha$ - and $\beta$-frequency bands further hints at the specificity of the effect. Importantly, studies indicate that $\gamma$-band synchronization may be a fundamental mechanism for the functional connection of neural populations in dynamic, transitory large-scale networks dedicated to specific tasks. Indeed, changes of $\gamma$-band neuronal oscillations clearly appear integral to a number of complex cognitive functions ranging from attention, language, memory and mental practice (Lutz et al., 2004; 
Bastiaansen and Hagoort, 2006) to crossmodal (Kanayama et al., 2009), and sensorimotor integration (Alonso et al., 1996; Wallace et al., 1996; Bauer et al., 2006; Szurhaj and Derambure, 2006). Relevant to the present study is that $\gamma$-band activity plays a specific role in first-hand pain processing (Chen and Herrmann, 2001; Ohara et al., 2006; Gross et al., 2007; Hauck et al., 2007). In particular, a recent MEG study showed that pain-induced $\gamma$-band oscillations in the primary somatosensory cortex were related to the subjective perception of pain stimulus intensity rather than to the objective intensity of the stimulus itself (Gross et al., 2007). Therefore, $\gamma$-band activity seems to reflect the neural processes underlying the representation of subjective aspects of perceptual (Gross et al., 2007) and cognitive (Jung-Beeman et al., 2004; Guggisberg et al., 2007; Sheth et al., 2009) experience. Our results extend the notion that $\gamma$-band activity is linked to higher order functions by indicating its relationship with the social sharing of pain. The increase of pain observation related $\gamma$-band synchronization of primary somatosensory motor cortical neurons, which are heavily connected at both anatomical and functional level (Rizzolatti and Luppino, 2001), hints at a specific role of these regions during observation of "flesh and bone" painful stimuli delivered to a model. While there is ample consensus that both the affective and the sensorimotor node of the pain matrix can be recruited during the vicarious experience of pain (Hein and Singer, 2008), we focused on the possible role of primary sensory and motor cortex in empathic pain mapping. Importantly, these regions seem to be specifically involved in the observation of flesh and bone pain stimuli delivered to body parts of stranger individuals (Avenanti et al., 2005, 2006, 2009; Bufalari et al., 2007; Fecteau et al., 2008; Minio-Paluello et al., 2006, 2009). The involvement of sensorimotor regions in the empathic mapping of others' pain is in keeping with TMS (Avenanti et al., 2005, 2006; Minio-Paluello et al., 2006), somatosensory (Bufalari et al., 2007) and laser-evoked potentials (Valeriani et al., 2008) studies. However, the main point of novelty of our study is that the essence of empathic mapping of observed pain resides in the dynamic cross-talk between somato-motor regions much more than on their separate neural activity. Indeed, mere observation of needle penetrating the hand of a stranger model induced a specific, fast and short-lasting increase in the functional connectivity between the primary somatosensory and motor cortex in the absence of detectable neural activity modulation in each area. The specific correlation between $\gamma$-band coherence with selfand other-oriented ratings of pain intensity and unpleasantness when observing needle-in hand clips but not when observing Q-tip on hand or static hand movie clips, also speaks in favor of a specific relationship between pain observation and functional sensorimotor coupling. The $\gamma$-band sensorimotor cross-talk underlying empathy for pain was not different when the observing subject performed an isometric contraction of the opponent muscle of the thumb, perceived non-painful electric stimuli on the skin overlaying the thumb opponent muscle, or was at rest, i.e., did not perform any task in addition to the pain observation one. This pattern of results indicates that the emergence of a

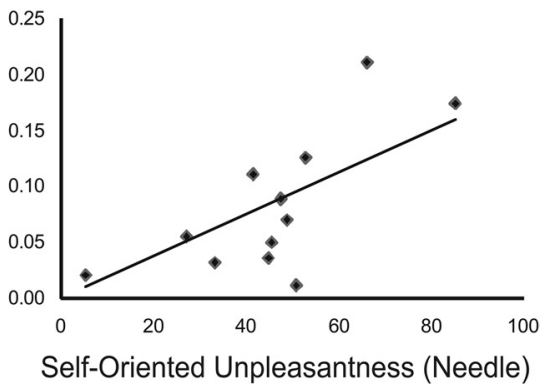

Self-Oriented Unpleasantness (Needle)

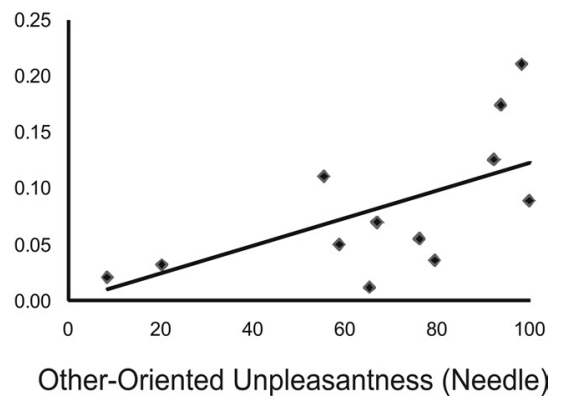

Other-Oriented Unpleasantness (Needle)

Figure 5. Correlational analysis. Scatter plots of sensorimotor coherence values in the Needle block and VAS scores concerning intensity and unpleasantness of the sensation derived from observation of others' pain, attributed to self (self-oriented) (top row) or to the model (other oriented) (bottom row).

dynamic sensorimotor network from mapping others' pain may be a powerful, automatic process that minimizes the interference of additional tasks.

\section{Sensory and motor cortical signatures of onlookers' resting state in $\alpha$ and $\beta$ frequency bands}

In the present study, interareal cross-talk as indexed by sensorimotor coherence was modulated by pain observation only in the $\gamma$-frequency band. Although no pain-observation related modulation of interareal cross-talk was found in any other bands, changes of activity in primary SI and MI cortices, as indexed by spectral power values, was found in $\alpha$ and $\beta$ frequency bands. However, such modulation did not depend on pain observation but on the onlookers' state. Indeed, we found that the spectral power, in both $\alpha$ - and $\beta$-frequency band, both for the SI and MI sources, was higher in the rest with respect to sensory and motor tasks. Spontaneous oscillatory activity is related to the functional state of a given system. Studies in the primary visual, somatosensory, and motor areas show that higher amplitude of oscillatory activity reflects resting states, whereas lower amplitude is associated with higher activation and excitability (Pfurtscheller and Lopes da Silva, 1999). This effect has been linked to the notion of “idling systems," i.e., systems which do not process any information at a given instant (Adrian and Matthews, 1934). In particular, while the occipital $\alpha$-rhythm reflects the idling condition of visual areas (Pfurtscheller and Lopes da Silva, 1999), the rolandic $\mu$ rhythm constitutes a signature of idling sensorimotor systems (Kuhlman, 1978). Mu rhythm is largely independent from the presence or absence of visual input and, occurring only when muscles are relaxed, is considered a motor-relaxation-associated rhythm (Buzsáki, 2006). Moreover, $\mu$ rhythm is thought to originate from the primary somatosensory cortex representing the hand, and in particular from the thumb (Hari and Salmelin, 1997). In view of this, our result of a rest specific increase of $\alpha$ and $\beta$ bands spectral power may reflect the idling status of the primary sensory and motor areas at rest. This status is likely interrupted by the stimulation of the thumb, occurring in the Sensory and motor tasks. Moreover, the significant increase of the senso- 
rimotor coherence found in the $\alpha$ band, as well as the trend effect shown in the $\beta$ band, is likely attributable to the high synchronized activity of the somatosensory system, characterized by a $\mu$ rhythm at rest.

All in all, the increase of cortical sensorimotor coherence contingent upon observation of noxious stimuli delivered to others may represent a neural signature of the interareal cross-talk that underpins the basic form of empathy we have called sensorimotor contagion (Avenanti et al., 2005). It is worth noting that this type of resonance seems to be absent in persons with autistic spectrum disorders (Minio-Paluello et al., 2009). Moreover, patients with autism exhibit patterns of aberrant $\gamma$-band EEG activity $(\sim 40 \mathrm{~Hz})$ contingent upon a visual binding task (Grice et al., 2001), a result which is in keeping with neuroimaging studies indicating that disrupted long-range connectivity and interregional interactions may underlie a range of behavioral deficits in autism (Müller, 2008; Uhlhaas and Singer, 2008). Thus, we posit that a lack of $\gamma$-band sensorimotor coherence increase during pain observation may represent a sensitive marker of empathic reactivity in a number of clinical conditions where this process is likely defective.

\section{References}

Adrian ED, Matthews BH (1934) The Berger rhythm: potential changes from the occipital lobes in man. Brain 57:355-385.

Albiero P, Ingoglia S, Lo Coco A (2006) Contributo all'adattamento italiano dell'Interpersonal Reactivity Index. TPM 13:107-125.

Alonso JM, Usrey WM, Reid RC (1996) Precisely correlated firing in cells of the lateral geniculate nucleus. Nature 383:815-819.

Avenanti A, Bueti D, Galati G, Aglioti SM (2005) Transcranial magnetic stimulation highlights the sensorimotor side of empathy for pain. Nat Neurosci 8:955-960.

Avenanti A, Minio Paluello I, Bufalari I, Aglioti SM (2006) Stimulus-driven modulation of motor-evoked potentials during observation of others' pain. Neuroimage 32:316-324.

Avenanti A, Minio-Paluello I, Bufalari I, Aglioti SM (2009) The pain of a model in the personality of an onlooker: influence of state-reactivity and personality traits on embodied empathy for pain. Neuroimage 44:275-283.

Avikainen S, Forss N, Hari R (2002) Modulated activation of the human SI and SII cortices during observation of hand action. Neuroimage 15:640-646.

Barbati G, Porcaro C, Zappasodi F, Rossini PM, Tecchio F (2004) Optimization of an independent component analysis approach for artifact identification and removal in magnetoencephalographic signals. Clin Neurophysiol 115:1220-1232.

Barbati G, Sigismondi R, Zappasodi F, Porcaro C, Graziadio S, Valente G, Balsi M, Rossini PM, Tecchio F (2006) Functional source separation from Magnetoencephalographic signals. Hum Brain Mapp 27:925-934.

Bastiaansen M, Hagoort P (2006) Oscillatory neuronal dynamics during language comprehension. Prog Brain Res 159:179-196.

Bauer M, Oostenveld R, Peeters M, Fries P (2006) Tactile spatial attention enhances $\gamma$-band activity in somatosensory cortex and reduces lowfrequency activity in parieto-occipital areas. J Neurosci 26:490-501.

Benuzzi F, Lui F, Duzzi D, Nichelli PF, Porro CA (2008) Does it look painful or disgusting? Ask your parietal and cingulate cortex. J Neurosci 28:923-931.

Bonino S, Lo Coco A, Tani F (1998) Empatia. I processi di condivisione delle emozioni. Firenze, Italy: Giunti.

Bufalari I, Aprile T, Avenanti A, Di Russo F, Aglioti SM (2007) Empathy for pain and touch in the human somatosensory cortex. Cereb Cortex 17:2553-2561.

Buzsáki G (2006) Rhythms of the brain. Oxford: Oxford UP.

Chen AC, Herrmann CS (2001) Perception of pain coincides with the spatial expansion of electroencephalographic dynamics in human subjects. Neurosci Lett 297:183-186.

Cheng Y, Lin CP, Liu HL, Hsu YY, Lim KE, Hung D, Decety J (2007) Expertise modulates the perception of pain in others. Curr Biol 17:1708-1713.

Cheng Y, Yang CY, Lin CP, Lee PL, Decety J (2008) The perception of pain in others suppresses somatosensory oscillations: a magnetoencephalography study. Neuroimage 40:1833-1840.

Costantini M, Galati G, Romani GL, Aglioti SM (2008) Empathic neural reactivity to noxious stimuli delivered to body parts and non-corporeal objects. Eur J Neurosci 28:1222-1230.

Davis MH (1996) Empathy: a social approch. Madison, WI: Westview.

Doesburg SM, Roggeveen AB, Kitajo K, Ward LM (2008) Large-scale gamma-band phase synchronization and selective attention. Cereb Cortex 18:386-396.

Eisenberger NI, Lieberman MD, Williams KD (2003) Does rejection hurt? An FMRI study of social exclusion. Science 302:290-292.

Engel AK, Singer W (2001) Temporal binding and the neural correlates of sensory awareness. Trends Cogn Sci 5:16-25.

Fecteau S, Pascual-Leone A, Théoret H (2008) Psychopathy and the mirror neuron system: preliminary findings from a non-psychiatric sample. Psychiatry Res 160:137-144.

Fries P (2005) A mechanism for cognitive dynamics: neuronal communication through neuronal coherence. Trends Cogn Sci 9:474-480.

Fries P (2009) Neuronal gamma-band synchronization as a fundamental process in cortical computation. Annu Rev Neurosci 32:209-224.

Grice SJ, Spratling MW, Karmiloff-Smith A, Halit H, Csibra G, de Haan M, Johnson MH (2001) Disordered visual processing and oscillatory brain activity in autism and Williams syndrome. Neuroreport 12:2697-2700.

Gross J, Schnitzler A, Timmermann L, Ploner M (2007) Gamma oscillations in human primary somatosensory cortex reflect pain perception. PLoS Biol 5:e133.

Guggisberg AG, Dalal SS, Findlay AM, Nagarajan SS (2007) High-frequency oscillations in distributed neural networks reveal the dynamics of human decision making. Front Hum Neurosci 1:14.

Hari R, Salmelin R (1997) Human cortical oscillations: a neuromagnetic view through the skull. Trends Neurosci 20:44-49.

Hauck M, Lorenz J, Engel AK (2007) Attention to painful stimulation enhances $\gamma$-band activity and synchronization in human sensorimotor cortex. J Neurosci 27:9270-9277.

Hein G, Singer T (2008) I feel how you feel but not always: the empathic brain and its modulation. Curr Opin Neurobiol 18:153-158.

Jasper H (1958) Report of the committee on methods of clinical examination in electroencephalography. Electroencephalogr Clin Neurophysiol 10:370-371.

Jensen O, Kaiser J, Lachaux JP (2007) Human gamma-frequency oscillations associated with attention and memory. Trends Neurosci 30:317-324.

Jung-Beeman M, Bowden EM, Haberman J, Frymiare JL, Arambel-Liu S, Greenblatt R, Reber PJ, Kounios J (2004) Neural activity when people solve verbal problems with insight. PLoS Biol 2:E97.

Kanayama N, Sato A, Ohira H (2009) The role of gamma band oscillations and synchrony on rubber hand illusion and crossmodal integration. Brain Cogn 69:19-29.

Kuhlman WN (1978) Functional topography of the human mu rhythm. Electroencephalogr Clin Neurophysiol 44:83-93.

Lamm C, Batson CD, Decety J (2007) The neural substrate of human empathy: effects of perspective-taking and cognitive appraisal. J Cogn Neurosci 19:42-58.

Lutz A, Greischar LL, Rawlings NB, Ricard M, Davidson RJ (2004) Longterm meditators self-induce high-amplitude gamma synchrony during mental practice. Proc Natl Acad Sci U S A 101:16369-16373.

Makeig S, Debener S, Onton J, Delorme A (2004) Mining event-related brain dynamics. Trends Cogn Sci 8:204-210.

Melzack R (1999) From the gate to the neuromatrix. Pain [Suppl 6]:S121-S126.

Minio-Paluello I, Avenanti A, Aglioti SM (2006) Left hemisphere dominance in reading the sensory qualities of others' pain? Soc Neurosci 1:320-333.

Minio-Paluello I, Baron-Cohen S, Avenanti A, Walsh V, Aglioti SM (2009) Absence of embodied empathy during pain observation in Asperger syndrome. Biol Psychiatry 65:55-62.

Morrison I, Lloyd D, di Pellegrino G, Roberts N (2004) Vicarious responses to pain in anterior cingulate cortex: is empathy a multisensory issue? Cogn Affect Behav Neurosci 4:270-278.

Müller RA (2008) From loci to networks and back again: anomalies in the study of autism. Ann N Y Acad Sci 1145:300-315.

Ohara S, Crone NE, Weiss N, Lenz FA (2006) Analysis of synchrony dem- 
onstrates 'pain networks' defined by rapidly switching, task-specific, functional connectivity between pain-related cortical structures. Pain 123:244-253.

Oldfield RC (1971) The assessment and analysis of handedness: the Edinburgh inventory. Neuropsychologia 9:97-113.

Pfurtscheller G, Lopes da Silva FH (1999) Event-related EEG/MEG synchronization and desynchronization: basic principles. Clin Neurophysiol 110:1842-1857.

Preston SD, de Waal FB (2002) Empathy: Its ultimate and proximate bases. Behav Brain Sci 25:1-20.

Rizzolatti G, Luppino G (2001) The cortical motor system. Neuron 31:889-901.

Rizzolatti G, Fogassi L, Gallese V (2001) Neurophysiological mechanism underlying the understanding and imitation of action. Nat Rev Neurosci 2:661-670.

Saarela MV, Hlushchuk Y, Williams AC, Schürmann M, Kalso E, Hari R (2007) The compassionate brain: humans detect intensity of pain from another's face. Cereb Cortex 17:230-237.

Schoffelen JM, Oostenveld R, Fries P (2008) Imaging the human motor system's beta-band synchronization during isometric contraction. Neuroimage 41:437-447.

Sheth BR, Sandkühler S, Bhattacharya J (2009) Posterior beta and anterior gamma oscillations predict cognitive insight. J Cogn Neurosci 21:12691279.

Silberstein P, Pogosyan A, Kühn AA, Hotton G, Tisch S, Kupsch A, DowseyLimousin P, Hariz MI, Brown P (2005) Cortico-cortical coupling in Parkinson's disease and its modulation by therapy. Brain 128:1277-1291.

Singer W, Gray CM (1995) Visual feature integration and the temporal correlation hypothesis. Annu Rev Neurosci 18:555-586.

Singer T, Seymour B, O’Doherty J, Kaube H, Dolan RJ, Frith CD (2004) Empathy for pain involves the affective but not sensory components of pain. Science 303:1157-1162.

Singer T, Seymour B, O’Doherty JP, Stephan KE, Dolan RJ, Frith CD (2006) Empathic neural responses are modulated by the perceived fairness of others. Nature 439:466-469.
Szurhaj W, Derambure P (2006) Intracerebral study of gamma oscillations in the human sensorimotor cortex. Prog Brain Res 159:297-310.

Tallon-Baudry C, Bertrand O (1999) Oscillatory gamma activity in humans and its role in object representation. Trends Cogn Sci 3:151-162.

Tecchio F, Porcaro C, Barbati G, Zappasodi F (2007) Functional source separation and hand cortical representation for a brain-computer interface feature extraction. J Physiol 580:703-721.

Tecchio F, Zappasodi F, Porcaro C, Barbati G, Assenza G, Salustri C, Rossini PM (2008) High-gamma band activity of primary hand cortical areas: a sensorimotor feedback efficiency index. Neuroimage 40:256-264.

Tracey I, Mantyh PW (2007) The cerebral signature for pain perception and its modulation. Neuron 55:377-391.

Uhlhaas PJ, Haenschel C, Nikolić D, Singer W (2008) The role of oscillations and synchrony in cortical networks and their putative relevance for the pathophysiology of schizophrenia. Schizophr Bull 34:927-943.

Valeriani M, Betti V, Le Pera D, De Armas L, Miliucci R, Restuccia D, Avenanti A, Aglioti SM (2008) Seeing the pain of others while being in pain: a laser-evoked potentials study. Neuroimage 40:1419-1428.

Varela F, Lachaux JP, Rodriguez E, Martinerie J (2001) The brainweb: phase synchronization and large-scale integration. Nat Rev Neurosci 2:229239.

Wallace MT, Wilkinson LK, Stein BE (1996) Representation and integration of multiple sensory inputs in primate superior colliculus. J Neurophysiol 76:1246-1266.

Womelsdorf T, Fries P (2006) Neuronal coherence during selective attentional processing and sensory-motor integration. J Physiol Paris 100:182193.

Young CK, McNaughton N (2009) Coupling of theta oscillations between anterior and posterior midline cortex and with the hippocampus in freely behaving rats. Cereb Cortex 19:24-40.

Zaki J, Ochsner KN, Hanelin J, Wager TD, Mackey SC (2007) Different circuits for different pain: patterns of functional connectivity reveal distinct networks for processing pain in self and others. Soc Neurosci 2:276-291. 